\title{
Emotive concept nouns and motor responses: Attraction or repulsion?
}

\author{
Laura Freina, Giulia Baroni, Anna M. Borghi, and Roberto Nicoletti \\ University of Bologna, Bologna, Italy
}

\begin{abstract}
We carried out three experiments aimed at testing whether hand posture affects the compatibility effect that Chen and Bargh (1999) found between a word's emotional connotation and arm movement direction. In the present study, participants responded by pressing two buttons: one placed near their body, the other far away. In Experiment 1, in which they pressed the response button with their hand open, RTs were shorter when participants pressed the far button for positive words and the near button for negative words, as if they simulated reaching for something good and avoiding something bad. However, in Experiments 2 and 3, in which participants pressed the response button with a tennis ball in their hand, RTs were shorter when participants pressed the near button for positive words and the far button for negative words, as if they simulated drawing a good thing closer and pushing a bad thing away. Results are discussed within the framework of theories on concept grounding in emotion and action systems.
\end{abstract}

In recent years, an embodied cognition approach has been gradually overtaking the traditional cognitive view in which the mind is seen as a device for manipulating arbitrary symbols. This new position, according to which our cognitive system is grounded in sensorimotor processes, is emerging in all fields that relate to cognitive sciencesfor example, philosophy (Clark, 1997; Prinz, 2002), neurosciences (Berthoz, 1997; Pulvermüller, 2003; Rizzolatti \& Arbib, 1998), and experimental psychology (Barsalou, 1999; Elsner \& Hommel, 2001; Glenberg, 1997). In addition, many computational models based on an embodied approach have been proposed (e.g., Elman et al., 1996; Nolfi \& Floreano, 2000).

According to the embodied theory, concepts consist of the reactivation of the same neural activation pattern that is present when we perceive and/or interact with the objects or entities they refer to (Barsalou, 1999; Borghi, 2005; Gallese \& Lakoff, 2005; Glenberg, 1997). In the same way, according to the embodied view, understanding language implies forming a mental "simulation" of what is described linguistically. This simulation is thought to entail the recruitment of the same neurons, which are activated regardless of whether one is directly experiencing or is encountering a description of a situation, an action, an emotion, an object, or an entity (Barsalou, 1999; Gallese \& Lakoff, 2005; Gibbs, 2003; Glenberg, 1997; MacWhinney, 1999; Zwaan, 2004).

In the last decade, a large body of evidence has been gathered in support of the simulation theory of language comprehension. Recent studies on sentence comprehension show that people form a sensorimotor simulation of the action or situation that a sentence describes by internally reproducing that action or situation (Buccino et al., 2005;
Glenberg \& Kaschak, 2002; Zwaan \& Taylor, 2006; see also Klatzky, McCloskey, Doherty, Pellegrino, \& Smith, 1987; Klatzky, Pellegrino, McCloskey, \& Doherty, 1989). Results obtained with different methods have shown clearly that language comprehension implies the activation of the motor system, and therefore these results support the simulation theory. However, it is still a matter of debate as to whether this simulation is necessary for comprehension or is due to motor imagery. The results leave open an additional question as to the extent to which this simulation is detailed.

Recent evidence suggests that this simulation is quite accurate. For example, behavioral and TMS studies have shown that the comprehension of sentences that refer to actions performed with different effectors (hands, feet, mouth) differentially influences a participant's response with the hand (keyboard), with the foot (pedal), and with the mouth (microphone) (Buccino et al., 2005; Scorolli \& Borghi, 2007). The sensitivity to the effectors reveals that the simulation run during the comprehension of an action sentence is rather detailed and that it also takes into account the means involved in a motor action. In addition, other studies indicate that simulation activated during the processing of a sentence that refers to an object's movement contains directional information. For example, participants were faster in responding that close the drawer made sense when the effector moved away from the body rather than when it moved toward it, whereas the opposite was true (the effector moved toward the body) in responding to a sentence such as open the drawer (Borreggine \& Kaschak, 2006; Glenberg \& Kaschak, 2002). The same sensitivity to directional aspects (upward vs. downward) was obtained when participants were required to decide whether nouns such as foot and head referred to parts of

G. Baroni, giulia.baroni4@unibo.it 
an object or to an entity, such as a horse (Borghi, Glenberg, \& Kaschak, 2004).

Along the same line, other evidence shows that the motor system and the evaluation of emotional terms are strictly interwoven. For example, learning the meaning of a word while we are performing a specific movement can cause us to evaluate that word in a positive or negative manner. Cacioppo, Priester, and Berntson (1993) demonstrated the effect of arm movement on the attitude that people have toward Chinese ideograms they did not know. When participants were required to pull their arm toward their body in order to respond (i.e., an approach movement), they tended to provide a better evaluation of the ideograms than when they were required to extend their arms away from their body (i.e., a repulsion movement).

In Chen and Bargh's (1999) study, participants were shown words, such as love and hate, that have a positive or negative emotive valence. The task was to respond to the word's emotive valence by pulling or pushing a lever. Reaction times (RTs) were quicker when there was congruence between the performed arm movement and the word valence (i.e., when participants had to pull something closer for positive words and to push something away for negative words). The same result was obtained when, instead of providing an evaluation, participants were asked to react to words appearing on a computer screen only by pushing or pulling the lever. Similar results were found with a noungeneration task. Participants were required to write the names of famous people and then to make an evaluation performing a movement that could be of approach or avoidance (Förster \& Strack, 1997). Results showed that people tended to produce names of famous people they liked or disliked congruently with the movement they made.

Although the reported evidence suggests that we activate simulations during sentence comprehension and that we recruit the motor system during the evaluation of emotional terms, the extent to which these simulations are specific is still a matter for debate. In the present study, we investigated the degree of specificity involved in these simulations. In particular, we aimed to understand whether reading a word with multiple meanings that have different positive versus negative emotional connotations differentially activates the motor system. More specifically, our hypothesis is that, if reading a positive or negative word activates a simulation of the object or entity to which it refers, this simulation should affect the motor response.

As we reported above, it has been demonstrated that positive stimuli tend to facilitate approach movements, whereas negative stimuli facilitate avoidance movements. Differing accounts of these effects have been proposed. According to the specific muscle activation account, the effects are caused by an association of extension movements with negative stimuli and of flexion movements with positive stimuli (Cacioppo et al., 1993). In line with this view, Tops and de Jong (2006) asked participants to respond to positive and negative words by pressing a response button with one hand while either holding a tennis ball in their other hand or keeping the other hand empty with fingers outstretched. Their findings showed that contracting the forearm flexors (i.e., holding the tennis ball) facilitated categorization of pleasant but not of unpleasant words in comparison with contracting the forearm tensors (i.e., stretching the fingers). However, their experiment does not deal with approach versus avoidance movements: The hand is kept steady while the other is used to make the choice. Their main concern is the psychological effect of contracting versus stretching specific muscles, whereas, in our experiments, we wanted to test whether the meaning assigned to a specific movement can change according to hand posture.

The distance-regulation account explains the results on the basis of the distance between the subject and the evaluated stimulus (Schneirla, 1959). The evaluative coding view instead attributes these effects to a correspondence relation between evaluative stimuli and responses on a cognitive, representational level rather than on the motor level. The embodied framework we adopt in the present article is in line with the evaluative approach in that it takes into account the flexible role played by context (Eder \& Rothermund, 2008). However, it differs from the evaluative approach in that it explains the effects on the basis of the reenactment of previous affective motor responses. For this reason, we consider how stimuli with the same valence are modulated, not only by the directional movements, but also by the hand posture used to respond to the stimuli. Our concern regards, in fact, the level of detail of the simulation and its interaction with the response movement. In particular, the question is whether hand posture can influence the meaning of such movement. Responses given with an open hand could induce people to simulate the action of reaching a positive object and avoiding a negative one. Participants should be quicker in moving the hand toward the stimulus when reading positive words and away from it when reading negative words. When participants are holding an object in the hand with which they respond, their responses could be coded in an opposite manner, inducing people to simulate the action of pushing away negative objects and drawing positive objects closer. This was the case in Chen and Bargh's (1999) experiments, in which participants responded by pushing or pulling a long lever.

We hypothesized that, if we found this pattern of results, it would show that the simulation run during word comprehension is very detailed. Specifically, such results would suggest that this simulation is sensitive not only to the effector involved and to directional aspects but also to the specific posture of the hand (e.g., closed vs. open; see Klatzky et al., 1987; Klatzky et al., 1989). Furthermore, we would demonstrate that a response movement is not interpreted as approach or avoidance in a unique manner but is defined mostly for its effect; that is, a movement could be coded as approach if it reduces the distance to a desirable object and as avoidance if it increases the distance from an undesirable object. In other words, the meaning assigned to the movement would be due to its goal rather than to kinematic aspects, such as the sequence of movements and their direction (Hommel, Müsseler, Aschersleben, \& Prinz, 2001).

\section{EXPERIMENT 1}

Chen and Bargh (1999) found that, in responding to positive words, people were quicker when they had to pull rather 
than push the lever. Likewise, for negative words, they were faster when they had to push rather than pull the lever. Chen and Bargh claimed that this result depended on the fact that, when reading a negative term, people activate their arm to make an avoidance movement (i.e., pushing away the negative object), whereas a positive word causes the opposite activation (i.e., pulling the positive object nearer).

If the simulations run during word comprehension were quite detailed, we predicted that the results would be influenced by hand posture. More specifically, we asked participants to respond by hitting, with the palm of their open right hand, one of two oversized buttons, one near and one far from the body. We predicted that, because participants responded with an empty hand, they would associate the arm movement no longer to the movement of an object, as was the case in Chen and Bargh's (1999) experiment, but to the action of grasping for or withdrawing from an object. Participants should therefore be quicker both in reaching the far button when reading a positive word, as they simulate reaching for the positive object, and in retracting their hand for negative words, as they simulate avoiding contact with a negative object. The results should therefore be the reverse of those obtained by Chen and Bargh. We expect, in fact, that arm extension can be interpreted as an approach and that arm flexion can be interpreted as an avoidance.

\section{Method}

Participants. Twenty-five University of Bologna students took part in a pilot study to select the stimuli; a separate group of 42 students took part in the experiment. Two participants were dismissed because they made too many errors, classifying more than $15 \%$ of the stimuli in a different manner from the normative one (e.g., a word that was classified as positive in the stimuli preparation process was later classified as negative). The 40 participants left were 17 men and 23 women, between 20 and 30 years old and with an average age of 23.33 years $(S D=2.39)$. All had normal or corrected-to-normal vision.

Stimuli. We selected the original 92 words used by Chen and Bargh (1999; see also Bargh, Chaiken, Govender, \& Pratto, 1992), translated them into Italian, and asked 25 University of Bologna students to evaluate them by answering a questionnaire. The words were collected from a sample population of 15 men and 10 women (average age $=23.08$ years; $S D=2.0$ ). For each word, the positive and negative valences were evaluated using separate Likert scales, both ranging from 1 to 4 . Then, using a method modeled on Bargh et al., which was based on Kaplan's (1972) work, we computed the following scores for each word:

1. A positive or negative evaluation, ranging from -3 to 3 , was defined as the average of all the differences between the positive and the negative scores. ${ }^{1}$ Words that obtained an overall evaluation ranging from -3 to 0 were classified as negative; the others were classified as positive.

2. A word-ambivalence evaluation, ranging from 0 to 6 , was defined as the average of all the differences between the sums of the positive and the negative scores and the absolute values of their differences.

Words with an evaluation score of between -2 and 2 were discarded, because they were not sufficiently emotionally charged. We also removed all words with an ambivalence score greater than 1.0, because, as demonstrated by Bargh et al. (1992), ambivalence strongly influences RTs. The 29 negative and 33 positive stimuli we obtained were reduced to a total of 58 stimuli. The 4 positive stimuli that presented the greatest $S D$ in their ambivalence score (i.e., those that were less homogeneously evaluated) were selected for the training session, together with 6 stimuli with an evaluation score of just below -2 or just above 2 . The training session consisted of the ran- dom presentation of 10 stimuli followed by the random presentation of 58 experimental stimuli. Each word was seen only once by each participant (a list of stimuli can be found at http://laral.istc.cnr.it/ borghi/freina-baroni-borghi-nicoletti-stimuli.htm).

Apparatus. A modified keyboard with only the space bar and two oversized buttons was used. The response buttons were labeled with the words POSITIVO and NEGATIVO, written in a large font so that the labels could be read easily. The keyboard was turned lengthwise, with the narrow part facing the participant so that one button was near the participant and the other was far away and closer to the computer screen; the space bar was between the two buttons. The experiment took place in a dimly lit noiseless room. Participants were seated facing a $17-$ in. CRT display driven by a $700-\mathrm{MHz}$ computer. E-Prime 1.1 software was used.

Procedure. Participants were asked to, as quickly as possible, classify each word as positive or negative by hitting the corresponding button with their right hand open. To start each trial, participants had to press the central part of the space bar, which forced the right hand into a central position. After the space bar was pressed, a cross appeared in the middle of the screen; after $500 \mathrm{msec}$, the cross was replaced by a word. The stimulus remained on the screen until the participant pressed either the positive or the negative button with an open hand. Participants received feedback for both correct and incorrect responses and pressed the space bar to start the next trial.

In the positive far-negative near (PosFar) condition, participants had to press the far button for positive words and the near button for negative words. In the positive near-negative far (PosNear) condition, the button assignments were reversed. Each participant was randomly assigned to the PosFar or PosNear condition. Therefore, participants assigned to each condition were selected from the same pool at around the same time and were treated in the same way. We predicted that the PosFar condition would produce faster RTs than those for the PosNear condition, since it was congruent with the grasping movement for positive words and with the avoidance movement for negative ones.

\section{Results and Discussion}

Errors, which amounted to $3.75 \%$ of the overall data, were not considered for the data analysis. An ANOVA on errors revealed that there was no speed-accuracy tradeoff: Neither the main effects of instruction and word valence nor the interaction between them were significant $(p=.19 ; p=.65 ; p=.65$, respectively); therefore, we focused on the RT analyses. To screen for outliers, scores that were $2 S D$ s higher or lower than the mean participant score were removed for each participant. This trimming method eliminated another $4.18 \%$ of the collected data. The remaining RTs were submitted to two mixed $2 \times 2$ ANOVAs. In the analysis with participants as the random factor $\left(F_{1}\right)$, the instruction factor (PosFar vs. PosNear) was manipulated between participants and the word valence factor (positive vs. negative) was manipulated within participants. With materials as the random factor $\left(F_{2}\right)$, the instruction factor (PosFar vs. PosNear) was manipulated within items and the word valence factor (positive vs. negative) was manipulated between items.

As predicted, the RTs were significantly faster in the PosFar than in the PosNear condition $\left[F_{1}(1,38)=5.94\right.$, $M S_{\mathrm{e}}=45,802.68, p<.020,836$ vs. $953 \mathrm{msec}$, respectively; $\left.F_{2}(1,56)=219.25, M S_{\mathrm{e}}=1,231.66, p<.001\right]$. Participants were faster at reaching than at withdrawing when they read a positive word and faster at withdrawing than at reaching when they processed a negative word. The interaction between instruction (PosFar vs. PosNear) and word valence (positive vs. negative) was also significant 


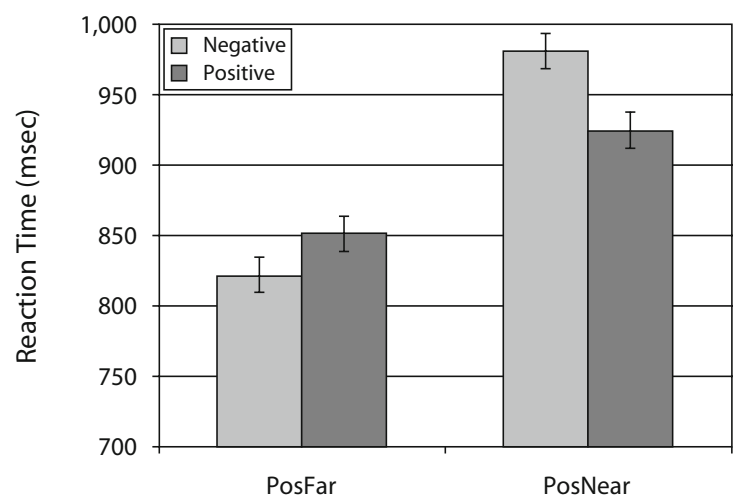

Instruction

Figure 1. Experiment 1. Interaction between instruction (PosFar vs. PosNear) and word valence (positive vs. negative) factors.

$\left[F_{1}(1,38)=17.10, M S_{\mathrm{e}}=2,152.42, p<.001 ; F_{2}(1,56)=\right.$ 43.39, $\left.M S_{\mathrm{e}}=1,231.66, p<.001\right]$. (See Figure 1.)

Because of the presence of a main effect of the instruction factor, we ran separate analyses, one for the positive and one for the negative terms. For both, we ran two ANOVAs: analysis of participants $\left(F_{1}\right)$ and materials $\left(F_{2}\right)$.

The instruction factor showed significant differences for negative stimuli: As we expected, RTs were faster in the PosFar than in the PosNear condition $\left[F_{1}(1,38)=\right.$ $11.51, M S_{\mathrm{e}}=22,094.76, p<.0016,822$ vs. $981 \mathrm{msec}$, respectively; $F_{2}(1,28)=402.14, M S_{\mathrm{e}}=700.94, p<.001$, 844 vs. $984 \mathrm{msec}$, respectively]. The analysis of positive stimuli showed that RTs were faster in the PosFar than in the PosNear condition, but the difference reached significance only in the analysis of materials $\left[F_{1}(1,38)=2.1\right.$, $M S_{\mathrm{e}}=25,860.43, p=.16,851$ vs. $925 \mathrm{msec}$, respectively; $F_{2}(1,28)=23.61, M S_{\mathrm{e}}=1,762.34, p<.001,875$ vs. $928 \mathrm{msec}$, respectively].

The results of Experiment 1 demonstrate that hand posture influences RTs. In Chen and Bargh's (1999) experiments, in which a lever was pushed or pulled, participants were faster at extending their arm when faced with a negative term, since arm extension is considered to be an avoidance movement, and faster at retracting their arm when processing a positive word, since arm retraction can be considered to be an attraction movement. In our experiment, in which participants were required to hit either a far or a near button with the open hand, the opposite results were obtained: People were faster at withdrawing from the negative object and at reaching for the positive object. So, the same movement of the arm can be interpreted as an avoidance or as an approach, depending on the response modality and the hand posture used (closed hand with lever vs. open empty hand).

\section{EXPERIMENT 2}

In Experiment 2, we investigated whether the different interpretation of arm movement we found comparing our Experiment 1 with that in Chen and Bargh (1999) was due to hand posture and not to other factors. Therefore, we designed an experiment that differed from Experiment 1 only in that participants had to hit the oversized buttons with a hand holding a tennis ball. We hypothesized that, with an object in their hand, participants could no longer interpret the movement as reaching for versus avoiding an object, but rather as pushing an object away versus pulling it closer.

More specifically, if participants are asked to hold a tennis ball while answering, we predicted the same results as those obtained in the study by Chen and Bargh (1999), even though their task implied moving a lever and ours implied moving a graspable object and placing it elsewhere. In other words, we predicted that, with an object in their hand, participants would associate the pressing of the far button with pushing an object away and the pressing of the near button with pulling an object closer.

\section{Method}

The only difference in apparatus and procedure from that used in Experiment 1 was that participants were asked to respond by hitting the response buttons with a tennis ball held firmly in their right hand. Stimuli were the same.

Participants. Forty-seven University of Bologna students who did not participate in Experiment 1 took part. Seven were eliminated because their classification errors exceeded the $15 \%$ threshold, leaving 40 participants (18 men, 22 women, $19-29$ years old, $M=23.13$, $S D=2.33$ ). All had normal or corrected-to-normal vision.

As in the previous experiment, in the PosFar condition, participants had to press the far button for positive words and the near button for negative words. In the PosNear condition, the button assignments were reversed. Each participant was randomly assigned to the PosFar or the PosNear condition.

\section{Results and Discussion}

Errors represented $4.35 \%$ of the overall data. An ANOVA on errors revealed that there were no main effects of instruction $(p=1.00)$ or word valence $(p=.41)$. Only the interaction between these two factors reached significance $(p<.04)$, probably due to the fact that, in the PosNear condition, negative items elicited less error than positive ones did. However, the Newman-Keuls post hoc test showed no significant differences. We then focused on the RT analyses, using the same trimming method as in Experiment 1. This led to the removal of another $3.92 \%$ of the data. The remaining RTs were submitted to two mixed $2 \times 2$ ANOVAs. In the analysis with participants as the random factor $\left(F_{1}\right)$, the instruction factor (PosFar vs. PosNear) was manipulated between participants, and the word valence factor (positive vs. negative) was manipulated within participants. In the ANOVA with materials as the random factor $\left(F_{2}\right)$, the instruction factor (PosFar vs. PosNear) was manipulated within items, and the word valence factor (positive vs. negative) was manipulated between items.

We obtained a significant difference between the positive and the negative word valence in the analysis with participants as the random factor $\left[F_{1}(1,38)=12.738, M S_{\mathrm{e}}=\right.$ $1,760.76, p<.001,968$ vs. $934 \mathrm{msec}$, respectively] but not in the analysis of materials $\left[F_{2}(1,56)=3.94, M S_{\mathrm{e}}=\right.$ $8,993.16, p=.052]$. There was a significant interaction between instruction (PosFar vs. PosNear) and word valence (positive vs. negative) $\left[F_{1}(1,38)=18.55, M S_{\mathrm{e}}=\right.$ 


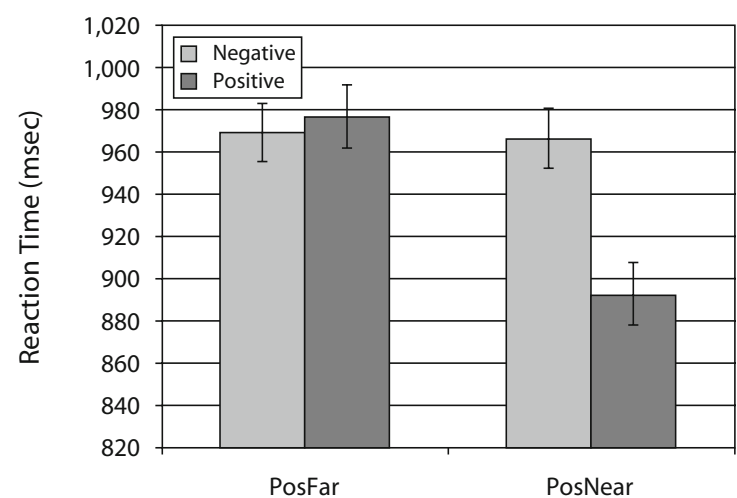

Instruction

Figure 2. Experiment 2. Interaction between instruction (PosFar vs. PosNear) and word valence (positive vs. negative) factors.

$1,760.76, p<.001 ; F_{2}(1,56)=21.05, M S_{\mathrm{e}}=1,740.72$, $p<.001]$. (See Figure 2.) In both analyses, the instruction factor did not reach significance.

For both positive and negative terms, two separate ANOVAs were performed, one with participants $\left(F_{1}\right)$ and one with materials $\left(F_{2}\right)$ as the random factor.

The instruction factor showed significant differences for positive stimuli, although this difference reached significance only in the analysis of materials, showing that RTs were slower in the PosFar than in the PosNear condition $\left[F_{1}(1,38)=2.007, M S_{\mathrm{e}}=34,613.94, p=.165\right.$, 976 vs. $893 \mathrm{msec}$, respectively; $F_{2}(1,28)=37.43, M S_{\mathrm{e}}=$ $2,209.03, p<.001,975$ vs. $899 \mathrm{msec}$, respectively]. For the negative stimuli, no difference was found $\left[F_{1}(1,38)=\right.$ $0.002, M S_{\mathrm{e}}=31,443.38, p=.94,969 \mathrm{msec}$ for the PosFar condition vs. $967 \mathrm{msec}$ for the PosNear one; $F_{2}(1,28)=$ $0.22, M S_{\mathrm{e}}=1,272.41, p=.64,974 \mathrm{msec}$ for the PosFar condition vs. $970 \mathrm{msec}$ for the PosNear one].

Although we found that RTs tended to be faster when participants had to move an object toward their body while processing positive words, results were not as predicted for negative stimuli, because, with negative words, we did not find faster RTs when people had to push the object in their hand away, which could have been caused by one of the following:

1. Pushing the response button with the tennis ball may have been associated with the action of hitting the object rather than moving it toward or away from the body, especially when the stimulus represented an object that could be squashed (e.g., spider).

2. There was a 500-msec delay between the hitting of the space bar (aimed at placing the hand in the middle between the two response buttons) and the stimulus onset. During Experiment 1, we observed that people tended to leave the hand on the space bar while waiting for the word to appear. In Experiment 2, participants hit the button with the ball and their hand bounced into the air. This could allow participants to move the hand freely; therefore, the hand position was not controlled at stimulus onset.

In Experiment 3, we addressed these two possibilities.

\section{EXPERIMENT 3}

This experiment replicates Experiment 2, with two changes to address the previously discussed points. We eliminated words that referred to objects that could be squashed and ran the experiment with a subset of the items selected previously. We also eliminated the $500-\mathrm{msec}$ delay between the hitting of the central space bar and the stimulus onset.

\section{Method}

We used the same apparatus as in Experiments 1 and 2.

Participants. Thirty-five University of Bologna students who had not participated in Experiment 1 or 2 took part. Three were discharged because their classification errors exceeded the $15 \%$ threshold, leaving 32 (11 men, 21 women; average age $=21.63$; $S D=2.03$ ). All had normal or corrected-to-normal vision; 3 were left-handed.

Stimuli. From the 58 stimuli used in the previous experiments, 18 were eliminated because they represented objects that could be squashed, as judged by at least 3 of the 4 native Italian speakers chosen to assess the stimuli. In order to maintain the same number of positive and negative terms, 2 further positive stimuli were eliminated. We chose 2 positive words for objects that 2 judges considered to be squashable. The remaining materials consisted of 44 words: 22 positive and 22 negative (see http://laral.istc.cnr.it/ borghi/freina-baroni-borghi-nicoletti-stimuli.htm). Each word was presented twice to each participant.

Procedure. The procedure was the same as in Experiment 2, except for the elimination of the 500-msec delay between the hitting of the central space bar and the stimulus onset.

As in the previous experiments, in the PosFar condition, participants had to press the far button for positive words and the near button for negative words. In the PosNear condition, the button assignments were reversed. Each participant was randomly assigned to the PosFar or PosNear condition.

\section{Results and Discussion}

Errors represented $4.90 \%$ of the overall data. An ANOVA on errors revealed that there was no speed-accuracy tradeoff. Neither the main effects of instruction and word valence nor the interaction between these two factors were significant ( $p=.56 ; p=.18 ; p=.43$, respectively), so we focused on the RT analyses. Using the trimming method used in the previous experiments eliminated another $4.87 \%$ of the data. The remaining responses were submitted to two mixed $2 \times 2$ ANOVAs. In the analysis with participants as the random factor $\left(F_{1}\right)$, the instruction factor (PosFar vs. PosNear) was manipulated between participants and the word valence factor (positive vs. negative) was manipulated within participants. In the ANOVA with materials as the random factor $\left(F_{2}\right)$, the instruction factor (PosFar vs. PosNear) was manipulated within items and the word valence factor (positive vs. negative) was manipulated between items.

As was predicted, the mean RT was significantly faster in the PosNear than in the PosFar condition $\left[F_{1}(1,30)=\right.$ $5.75, M S_{\mathrm{e}}=16,637.61, p<.023,949$ vs. $872 \mathrm{msec}$, respectively; $F_{2}(1,42)=165.66, M S_{\mathrm{e}}=817.86, p<.001$, 950 vs. $872 \mathrm{msec}$, respectively]. Participants were faster at pulling the tennis ball toward themselves when they read a positive word and faster at pushing it away when they processed a negative word. The interaction between instruc- 


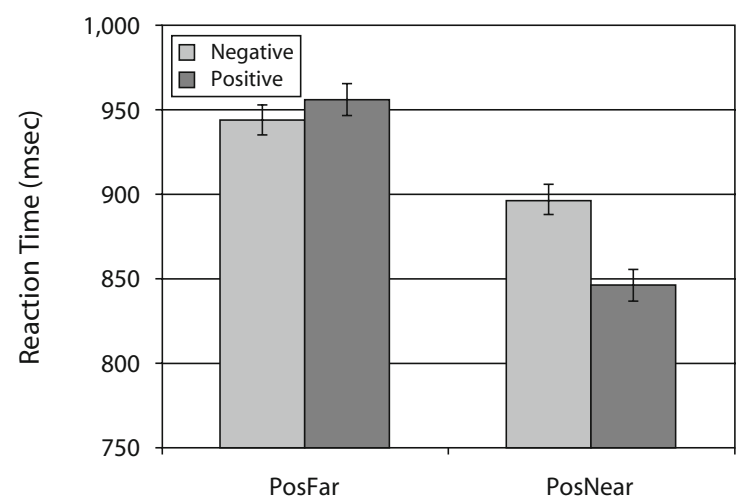

Instruction

Figure 3. Experiment 3. Interaction between instruction (PosFar vs. PosNear) and word valence (positive vs. negative) factors.

tion (PosFar vs. PosNear) and word valence (positive vs. negative) was also significant $\left[F_{1}(1,30)=7.96, M S_{\mathrm{e}}=\right.$ $1,857.09, p<.008 ; F_{2}(1,42)=26.71, M S_{\mathrm{e}}=817.86, p<$ .001]. (See Figure 3.)

Because of the presence of a main effect of the instruction factor, we ran separate analyses for the positive and for the negative terms, in order to determine whether the effect was present for both kinds of stimuli. In both cases, we ran two ANOVAs: analyses of participants $\left(F_{1}\right)$ and materials $\left(F_{2}\right)$.

The instruction factor showed significant differences for positive stimuli: As we expected, RTs were slower in the PosFar than in the PosNear condition $\left[F_{1}(1,30)=\right.$ $11.50, M S_{\mathrm{e}}=8,064.16, p<.002,953$ vs. $846 \mathrm{msec}$, respectively; $F_{2}(1,21)=163.53, M S_{\mathrm{e}}=813.69, p<.001$, 956 vs. $846 \mathrm{msec}$, respectively]. Also, the analysis of negative stimuli showed that RTs were slower in the PosFar than in the PosNear condition, but the difference reached significance only in the analysis of materials $\left[F_{1}(1,30)=\right.$ $1.69, M S_{\mathrm{e}}=10,430.36, p=.20 ; F_{2}(1,21)=29.51, M S_{\mathrm{e}}=$ $822.03, p<.001,944$ vs. $897 \mathrm{msec}$, respectively].

The results of Experiment 3 demonstrated that hand posture influences RTs. Whereas, in Experiment 1, in which participants were required to hit either a far or a near button with an open hand, people were faster at withdrawing from the negative object and reaching for the positive one, in this experiment opposite results were obtained: People were faster at pushing the ball away when reading a negative word and pulling it toward themselves when reading a positive word. Therefore, we can claim that arm flexion can be interpreted as an avoidance movement when participants respond with the open and empty hand and as an approach movement when participants are asked to respond while grasping a tennis ball. The opposite is true for arm extension.

\section{GENERAL DISCUSSION}

This study clearly shows that motor responses to emotive concept words are influenced strongly by hand posture. Our results not only support theories claiming that word comprehension activates a simulation, they help to specify the characteristics of this simulation (Gallese \& Goldman, 1998; Zwaan, 2004). That is, they suggest that the simulation run during word comprehension and evaluation is quite detailed, because different hand postures (open vs. closed hand) led to the assignment of an opposite meaning of approach-avoidance to the same movement (arm flexion vs. extension).

In our three experiments, participants had to classify words as positive or negative by pressing one of two buttons, one near and one far from the body. The movements required were similar to those of Chen and Bargh's (1999) experiments (i.e., arm flexion when pressing the near button and arm extension when pressing the far button). Unlike Chen and Bargh, we focused on the hand posture with which participants were asked to respond. In Experiment 1, participants pressed the response buttons with their right hand open. Results showed that they simulated the movement of reaching for something good (approach) or avoiding something bad (avoidance). Therefore, although the movement performed was the same as in Chen and Bargh, the results we obtained were the opposite. In two further experiments, we asked participants to push the response buttons with their right hand, in which they held a tennis ball. We found opposite results with respect to Experiment 1, although the results were similar to those of Chen and Bargh. The present results suggest that, if they held an object in their hand, participants simulated the actions of pushing away something bad (avoidance) and drawing in something good (approach). Results were stronger with stimuli that represented something that could not be squashed (e.g., unlike spider). The results are straightforward: The fact that in a couple of cases the difference was significant in the analysis with items, but not with participants, as the random factor simply reveals that there is some variability across participants, probably due to the fact that the mapping of valence to arm motions depends on the participants' construal of the task.

Taken together, our experiments show that hand posture plays a crucial role in determining the approach-avoidance meaning of response movements. This result helps to confute some theoretical accounts of approach-avoidance movements. In particular, theories based on a specific muscle activation account (see Cacioppo et al., 1993; Tops \& de Jong, 2006) or on evaluative aspects (see Eder \& Rothermund, 2008) could hardly explain our results. Our data clearly depend on a combination of motor and conceptual aspects and on a reenactment of previous affective motor responses. For this reason, the embodied account seems to be the best framework to explain our data, and the simulation theory leads to a better explanation for the different meanings that the participants assigned to the same movement.

Overall, we believe that our results also have important implications for theories on the relationship between language comprehension and the motor system that assigns relevance to goals for action representation. Specifically, they reveal that participants are sensitive to very fine aspects of hand postures, such as responding with an open hand in comparison with when the hand is holding an object. At a theoretical level, this suggests that the simula- 
tion run during comprehension is not only sensitive to the action goals (oriented to elements that are external to the participant's body) but also to kinematics aspects (i.e., to sensory codes coming from the participant's own body, representing fine-grained aspects such as those related to hand posture). Importantly, however, this happens when the sensitivity to fine-grained aspects related to hand posture influences the more general action goal and induces the participant to assign a different meaning to the whole movement (Hommel et al., 2001).

\section{AUTHOR NOTE}

We thank the EMCO (www.emco.unibo.it) and ROSSI (www .rossiproject.eu) groups for helpful comments on this work. This work was supported by MIUR (Ministero Italiano dell'Istruzione, dell'Università e della Ricerca) and by European Community, in Project ROSSI: Emergence of Communication in Robots Through Sensorimotor and Social Interaction (Grant Agreement No. 216125). Address correspondence to G. Baroni, Dipartimento di Discipline della Comunicazione, Via Azzo Gardino 23-40122 Bologna, Italy (e-mail: giulia.baroni4@unibo.it).

\section{REFERENCES}

Bargh, J. A., Chaiken, S., Govender, R., \& Pratto, F. (1992). The generality of the automatic attitude activation effect. Journal of Personality \& Social Psychology, 62, 893-912. doi:10.1037/0022 $-3514.62 .6 .893$

Barsalou, L. W. (1999). Perceptual symbol systems. Behavioral \& Brain Sciences, 4, 577-660. doi:10.1017/S0140525X99002149

Berthoz, A. (1997). Le sens du mouvement. Paris: Odile Jacob.

Borghi, A. M. (2005). Object concepts and action. In D. Pecher \& R. A. Zwaan (Eds.), The grounding of cognition: The role of perception and action in memory, language and thinking (pp. 8-34). Cambridge: Cambridge University Press.

Borghi, A. M., Glenberg, A. M., \& KaschaK, M. P. (2004). Putting words in perspective. Memory \& Cognition, 32, 863-873.

Borreggine, K. L., \& KaschaK, M. P. (2006). The action-sentence compatibility effect: It's all in the timing. Cognitive Science, 30, 10971112. doi:10.1207/s15516709 $\operatorname{cog} 0000 \_91$

Buccino, G., Riggio, L., Melli, G., Binkofski, F., Gallese, V., \& Rizzolatti, G. (2005). Listening to action-related sentences modulates the activity of the motor system: A combined TMS and behavioral study. Cognitive Brain Research, 24, 355-363. doi:10.1016/j .cogbrainres.2005.02.020

Cacioppo, J. T., Priester, J. R., \& Berntson, G. G. (1993). Rudimentary determinants of attitudes: II. Arm flexion and extension have differential effects on attitudes. Journal of Personality \& Social Psychology, 65, 5-17.

ChEN, M., \& BARGH, J. A. (1999). Consequences of automatic evaluation: Immediate behavioral predispositions to approach or avoid the stimulus. Personality \& Social Psychology Bulletin, 25, 215-224. doi:10.1177/ 0146167299025002007.

Clark, A. (1997). Being there: Putting brain, body, and world together again. Cambridge, MA: MIT Press.

Eder, A. B., \& Rothermund, K. (2008). When do motor behaviors (mis)match affective stimuli? An evaluative coding view of approach and avoidance reactions. Journal of Experimental Psychology: General, 137, 262-281. doi:10.1037/0096-3445.137.2.262

Elman, J. L., Bates, E. A., Johnson, M. H., Karmiloff-Smith, A., Parisi, D., \& Plunkett, K. (1996). Rethinking innateness: A connectionist perspective on development. Cambridge, MA: MIT Press.

ElsNer, B., \& Hommel, B. (2001). Effect anticipation and action control. Journal of Experimental Psychology: Human Perception \& Performance, 27, 229-240.

FÖRSTER, J., \& STRACK, F. (1997). Motor actions in retrieval of valenced information: A motor congruence effect. Perceptual \& Motor Skills, 85, 1419-1427.

Gallese, V., \& Goldman, A. (1998). Mirror neurons and the simulation theory of mind-reading. Trends in Cognitive Sciences, 2, 493-501.
Gallese, V., \& LAKOFF, G. (2005). The brain's concepts: The role of the sensory-motor system in conceptual knowledge. Cognitive Neuropsychology, 22, 455-479. doi:10.1080/02643290442000310

GIBBS, R. W., JR. (2003). Embodied experience and linguistic meaning. Brain \& Language, 84, 1-15.

GLENBERG, A. M. (1997). What memory is for. Behavioral \& Brain Sciences, 20, 1-55. doi:10.1017/S0140525X97000010

Glenberg, A. M., \& KaschaK, M. P. (2002). Grounding language in action. Psychonomic Bulletin \& Review, 9, 558-565.

Hommel, B., Müsseler, J., Aschersleben, G., \& Prinz, W. (2001). The theory of event coding (TEC): A framework for perception and action planning. Behavioral \& Brain Sciences, 24, 849-937.

KAPLAN, K. J. (1972). On the ambivalence-indifference problem in attitude theory and measurement: A suggested modification of the semantic differential technique. Psychological Bulletin, 77, 361-372.

Klatzky, R. L., McCloskey, B. P., Doherty, S., Pellegrino, J. W., $\&$ SмiтH, T. (1987). Knowledge about hand shaping and knowledge about objects. Journal of Motor Behavior, 19, 187-213.

Klatzky, R. L., Pellegrino, J. W., McCloskey, B. P., \& Doherty, S. (1989). Can you squeeze a tomato? The role of motor representations in semantic sensibility judgments. Journal of Memory \& Language, 28, 56-77. doi:10.1016/0749-596X(89)90028-4

MacWhinney, B. (1999). The emergence of language from embodiment. In B. MacWhinney (Ed.), The emergence of language (pp. 213256). Mahwah, NJ: Erlbaum.

Nolfi, S., \& Floreano, D. (2000). Evolutionary robotics. The biology, intelligence, and technology of self-organizing machines. Cambridge, MA: MIT Press.

PRINZ, J. J. (2002). Furnishing the mind: Concepts and their perceptual basis. Cambridge, MA: MIT Press.

PUlVERMüLleR, F. (2003). The neuroscience of language. On brain circuits of words and serial order. Cambridge: Cambridge University Press.

Rizzolatti, G., \& Arbib, M. A. (1998). Language within our grasp. Trends in Neurosciences, 21, 188-194.

Schneirla, T. C. (1959). An evolutionary and developmental theory of biphasic processes underlying approach and withdrawal. In M. R. Jones (Ed.), Nebraska Symposium on Motivation (Vol. 7, pp. 1-42). Lincoln: University of Nebraska Press.

Scorolli, C., \& Borghi, A. M. (2007). Sentence comprehension and action: Effector specific modulation of the motor system. Brain Research, 1130, 119-124. doi:10.1016/j.brainres.2006.10.033

Tops, M., \& DE Jong, R. (2006). Posing for success: Clenching a fist facilitates approach. Psychonomic Bulletin \& Review, 13, 229-234.

ZWAAN, R. A. (2004). The immersed experiencer: Toward an embodied theory of language comprehension. In B. H. Ross (Ed.), Psychology of learning and motivation (Vol. 44, pp. 35-62). San Diego: Academic Press.

ZWAan, R. A., \& TAYLOR, L. J. (2006). Seeing, acting, understanding: Motor resonance in language comprehension. Journal of Experimental Psychology: General, 135, 1-11.

\section{NOTE}

1. Bargh et al. (1992) computed a normative evaluation of each word separately, on an 11-point scale ranging from -5 to 5 , and used this single rating to compute the mean rating by sample. We needed a similar rating, but we collected only one series of questionnaires; therefore, we used these data to compute it. Each subject had given each word both a positive and a negative evaluation. Starting from these evaluations, we computed for each subject and for each word the rating as the difference between its positive and its negative score. This rating could range from 3 (if the word was evaluated as 4 on the positive scale and 1 on the negative scale) to -3 (if it was evaluated 1 on the positive scale and 4 on the negative scale). We then computed the average across subjects for each word. The goal of this kind of rating was to order the words and select those that had the higher scores as positive and the ones with the lower scores as negative.

(Manuscript received May 14, 2008; revision accepted for publication January 12, 2009.) 